\title{
Erratum to: In vitro digestion of starches in a dynamic gastrointestinal model: an innovative study to optimize dietary management of patients with hepatic glycogen storage diseases
}

\author{
Tatiéle Nalin $^{1,2}$ - Koen Venema ${ }^{3,9}$ - David A. Weinstein ${ }^{4}$ Carolina F. M de Souza ${ }^{2}$. \\ Ingrid D. S. Perry ${ }^{5,6}$ • Mario T. R. van Wandelen ${ }^{3} \cdot$ Margreet van Rijn $^{7}$ • \\ G. Peter A. Smit ${ }^{7}$ Ida V. D. Schwartz ${ }^{1,2,8}$ - Terry G. J. Derks ${ }^{7}$
}

Published online: 20 May 2015

(C) SSIEM 2015

Erratum to: J Inherit Metab Dis DOI 10.1007/s10545-014-9763-y

The name of Koen Venema is not correctly stated.

It must read Koen Venema, and K. Venema

The online version of the original article can be found at 10.1007/s10545014-9763-y.

Terry G. J. Derks

t.g.j.derks@umcg.nl

1 Post-Graduation Program in Genetics and Molecular Biology, Universidade Federal do Rio Grande do Sul, Porto Alegre, Brazil

2 Medical Genetics Service, Hospital de Clínicas de Porto Alegre, Porto Alegre, Brazil

3 The Netherlands Organization for Applied Scientific Research (TNO), Delft, The Netherlands

4 Glycogen Storage Disease Program, Division of Pediatric Endocrinology, Department of Pediatrics, University of Florida, Gainesville, Florida, USA

5 Food and Nutrition Research Centre, Hospital de Clínicas de Porto Alegre, Porto Alegre, Brazil

6 Health Unit, Universidade do Extremo Sul Criciúma, Criciúma, Brazil

7 Section of Metabolic Diseases, Beatrix Children's Hospital, University Medical Center of Groningen, University of Groningen, Hanzeplein 1, 9700, RB Groningen, The Netherlands

8 Department of Genetics, Universidade Federal do Porto Alegre, Rio Grande do Sul, Brazil

9 Present address: Beneficial Microbes Consultancy, Wageningen, The Netherlands 